\title{
A Review of the Research Status of Aflatoxin in Peanuts
}

\author{
Ruiyang Xu, Yu Liu, Yifan Jin \\ Chengdu University of Technology, Chengdu, China \\ Email: 1397012895@qq.com
}

How to cite this paper: Xu, R.Y., Liu, Y. and Jin, Y.F. (2021) A Review of the Research Status of Aflatoxin in Peanuts. Open Access Library Journal, 8: e7947. https://doi.org/10.4236/oalib.1107947

Received: September 10, 2021

Accepted: September 27, 2021

Published: September 30, 2021

Copyright () 2021 by author(s) and Open Access Library Inc.

This work is licensed under the Creative Commons Attribution International License (CC BY 4.0).

http://creativecommons.org/licenses/by/4.0/ (c) (i) Open Access

\begin{abstract}
Food safety is an important part of the healthy safety system and is closely related to people's health. Peanut is an important daily food and oil product in China. It is very important to ensure its safety, but peanuts are easily contaminated by aflatoxin. How to efficiently detect aflatoxin in peanut is particularly important. This paper reviews the related research literature of aflatoxin in peanuts at home and abroad in recent years, discusses its sources, hazards and detection methods. The advantages of laser-induced fluorescence detection technology were discussed, and some suggestions for the future detection of aflatoxin in peanut were put forward.
\end{abstract}

\section{Subject Areas}

Food Science \& Technology

\section{Keywords}

Aflatoxin, Peanut, Detection Technology, Laser-Induced Fluorescence

\section{1. 引言}

花生作为一种重要的粮油产品, 不止是中国为数不多具有明显竞争优势 的出口外汇农产品, 给我国带来大笔出口外汇收入, 对于国内民众, 花生、 花生制品也是一种不可或缺的日常食品 [1]。正因为其多油性, 花生在生长、 储存和食品加工过程中很容易被菌类污染：曲霉菌等真菌容易附着在花生外 壳和红衣上, 甚至是某些花生仁的胚芽和两片子叶中空的地方, 而曲霉在生 长代谢过程中会产生黄曲霰素(AFT), 污染花生; 被污染的花生用于榨油, 由 于黄曲霉毒素具有脂溶性，又很容易残留在花生油中 [1] [2] [3]。该毒素对生 物体具有很强的致癌性、致畸性，从而导致被污染花生、花生制品的不可食 用性，造成巨大的经济损失 [4]。由此，如何实现高效检测花生中的黄曲霉素， 
并做出一定防护措施就显得尤其重要。

\section{AFT来源与限定标准}

黄曲霉和寄生曲霉作为产生黄曲霉素的主要菌株, 通常寄生于油类产品, 如花生、大米等, 在生长代谢过程中, 会产生黄曲霉素(AFT)。黄曲霉毒素在 长波紫外光下产生荧光, 根据荧光颜色、 $\mathrm{RF}$ 值及结构等分别命名为 $\mathrm{B}_{1}, \mathrm{~B}_{2}$, $G_{1}, G_{2}, M_{1}, M_{2}, P_{1}, R_{1}, G M$ 和毒醇, 其中 $A_{1} B_{1}$ 和 $A F B_{2}$ 发出蓝色苂光, $\mathrm{AFG}_{1}$ 和 $\mathrm{AFG}_{2}$ 发出绿色荧光 [5] [6] [7]。作为一种具有相似结构的次生代谢产 物, AFT 都是二氢呋喃香豆素的衍生物, 其化学结构式如图 1,

自然环境下，在被污染的食品中只检测出 $\mathrm{AFB}_{1} 、 \mathrm{AFB}_{2} 、 \mathrm{AFG}_{1} 、 \mathrm{AFG}_{2}$ 、 $\mathrm{AFM}_{1}$ 和 $\mathrm{AFM}_{2}$, 其中以 $\mathrm{AFB}_{1}$ 存在量最大也毒性最高, $\mathrm{AFB}_{1}$ 的毒性约为 $\mathrm{KCN}$ 的 10 倍, 砒霜的 68 倍, 致癌性为二甲基亚硝胺的 75 倍, 奶油黄的 900 倍, 4-苯并萠的 4000 倍, 人畜误食该毒素能够引起内脏出血性坏死, 慢性中毒及 引发癌症[3]。 $\mathrm{AFM}_{1}$ 是 $\mathrm{AFB}_{1}$ 的代谢产物, 毒性仅次于 $\mathrm{AFB}_{1}$ 。

黄曲霉毒素的各种代谢产物的毒性强弱顺序是: $\mathrm{AFB}_{1}>\mathrm{AFM}_{1}>\mathrm{AFG}_{1}>$ $\mathrm{AFB}_{2}>\mathrm{AFM}_{2}>\mathrm{AFG}_{2}$ 。从毒性顺序可以看出, 结构中双呋喃环的末端具有双 键结构的毒性大, 不具双键结构的毒性相对较小 [8]。早在 1993 年世界卫生组 织就将 AFT 列为 “I 级致癌物质” , 世界各国都非常重视 AFT 的污染情况, 对其有严格的限量规定。

如表 1 所示, 不同国家针对食品中 AFT 的限量标准有所不同, 主要是限 制 AFT 中毒性最强的 $\mathrm{B}_{1}$ 和总 AFT 的含量。我国对于花生及花生制品的黄曲 霉素的控制标准主要在于 $\mathrm{AFTB}_{1}$, 即最高限量为 $20 \mu \mathrm{g} / \mathrm{kg}$ [5]。<smiles>COc1cc2c3c4c1C(=C(C(=O)CC4)C(O)O2)C1OC=CC3O1</smiles>

黄曲霉毒素B<smiles>COc1cc2c(c3oc(=O)c4c(c13)CCC4=O)C1(O)C=COC1O2</smiles>

黄曲霉毒素 $M_{1}$<smiles>COc1cc2c(c3oc(=O)c4c(c13)CCC4O)C1CCOC1O2</smiles>

黄曲霉毒素B $\mathrm{B}_{2}$<smiles>COc1cc2c(c3oc(=O)c4c(c13)CCC4=O)C1(O)CCOC1O2</smiles>

黄曲霉毒素 $\mathrm{M}_{2}$

图 1. 常见黄曲霉素的结构式 
表 1. 不同国家的黄曲霉素限定标准

\begin{tabular}{|c|c|c|c|c|c|c|c|c|}
\hline \multirow{2}{*}{ 国家和地区 } & \multirow{2}{*}{ 食品 } & \multirow{2}{*}{ 应用对象 } & \multicolumn{6}{|c|}{ 控制对象(最高限量)/( $\left.\mu \mathrm{g} \cdot \mathrm{kg}^{-1}\right)$} \\
\hline & & & 黄曲霘素总量 & $\mathrm{AFB}_{1}$ & $\mathrm{AFB}_{2}$ & $\mathrm{AFG}_{1}$ & $\mathrm{AFG}_{2}$ & $\mathrm{AFM}_{1}$ \\
\hline \multirow{6}{*}{ 中国 } & \multirow{2}{*}{ 花生及其制品 } & 直接供人食用的 & - & 20 & - & - & - & - \\
\hline & & 食用前经过物理处理的 & - & 20 & - & - & - & - \\
\hline & \multirow{2}{*}{ 坚果干制水果 } & 直接供人食用或作为食品成分 & - & - & - & - & - & - \\
\hline & & 食用前经过处理的 & - & -- & - & - & - & - \\
\hline & \multirow{2}{*}{ 奶、奶制品谷类 } & & - & - & - & - & - & 0.5 \\
\hline & & 直接供人类食用或作为食品成分 & - & -- & - & - & - & - \\
\hline \multirow{6}{*}{ 美国 } & \multirow{2}{*}{ 花生及其制品 } & 直接供人食用的 & 20 & - & - & - & - & - \\
\hline & & 食用前经过物理处理的 & 20 & - & - & - & - & - \\
\hline & \multirow{2}{*}{ 坚果干制水果 } & 直接供人食用或作为食品成分 & 20 & - & - & - & - & - \\
\hline & & 食用前经过处理的 & 20 & - & - & - & - & - \\
\hline & \multirow{2}{*}{ 奶、奶制品谷类 } & & - & - & - & - & - & 0.5 \\
\hline & & 直接供人类食用或作为食品成分 & - & - & - & - & - & - \\
\hline \multirow{6}{*}{ 日本 } & \multirow{2}{*}{ 花生及其制品 } & 直接供人食用的 & 10 & - & - & - & - & - \\
\hline & & 食用前经过物理处理的 & 10 & - & - & - & - & - \\
\hline & \multirow{2}{*}{ 坚果干制水果 } & 直接供人食用或作为食品成分 & 10 & - & - & - & - & - \\
\hline & & 食用前经过处理的 & 10 & - & - & - & - & - \\
\hline & \multirow{2}{*}{ 奶、奶制品谷类 } & & 10 & - & - & - & - & - \\
\hline & & 直接供人类食用或作为食品成分 & 10 & - & - & - & - & - \\
\hline \multirow{6}{*}{ 欧盟 } & \multirow{2}{*}{ 花生及其制品 } & 直接供人食用的 & 4 & 2 & - & - & - & - \\
\hline & & 食用前经过物理处理的 & 15 & 8 & - & - & - & - \\
\hline & \multirow{2}{*}{ 坚果干制水果 } & 直接供人食用或作为食品成分 & 4 & 2 & - & - & - & - \\
\hline & & 食用前经过处理的 & 10 & 5 & - & - & - & - \\
\hline & \multirow{2}{*}{ 奶、奶制品谷类 } & & - & - & - & - & - & 0.05 \\
\hline & & 直接供人类食用或作为食品成分 & 4 & 2 & - & - & - & - \\
\hline
\end{tabular}

\section{3. 危害}

之所以严格限制黄曲霉素的含量, 是因为黄曲霉毒素不止有很强的急性 毒性, 还有显著的慢性毒性: 黄曲霉素被认为是致癌性最强的化学致癌物之 一，在临床试验中发现，黄曲霉毒素主要损害肝脏，表现为肝细胞核肿胀、 脂肪变性、出血、坏死及胆管上皮、纤维组织增生, 导致禽类、灵长类和鱼 类等试验动物出现诱发试验性肝癌, 同时肾脏也可受损害 [9] [10]。

黄曲霉素对人与动物健康的危害都跟黄曲霉素抑制蛋白质的合成有很密 切的关系, 其分子中双呋喃环结构, 是产生毒性的重要结构[11]。Clifford 等 研究[12]发现, 黄曲霉素的细胞毒害作用, 是先干扰 DNA 与 mRNA 的合成, 进而再干扰蛋白质的合成，影响细胞代谢，最终导致机体全身性的损害。同 时，黄曲霉素产生的毒性还会通过母体遗传到子体中，进而对子体在胚胎期 
的免疫系统发育造成危害, 导致子体的免疫功能收到危害。

\section{4. 检测方法}

目前, 对于黄曲霉素的传统检测方法包括: 薄层分析法(TLC)、液相色谱 法(HPLC)、酶联免疫法(ELISA)、毛细管电泳法(CE)、荧光光谱法(IAC/SFB)、 金标试纸法、生物传感器法[13]-[23]。

TLC 法是检测黄曲霉素最为经典的方法, 也是一种国标方法。其原理是 针对不同的试样, 用适宜的萃取溶剂将黄曲霉素从试样中萃取出来, 经柱层 析净化后, 再在薄板上展开后分离。利用黄曲霉素的苂光特性, 根据苂光斑 点的强弱与标准比较确定其含量, 对于一些组分很复杂的试样要双向展开, 才能获得较高的灵敏度。TLC 法设备简单, 检测费用低, 但操作繁琐、费时, 萃取和净化效果不理想, 灵敏度差, 对操作人员的身体健康存在较大程度的 危害。

HPLC 法是近年来发展起来的一种检测方法。其原理是在高效液相色谱 仪上添加柱后衍生系统分离, 再用荧光检测器测定。当前, 该方法大多用免 疫亲和柱来净化、分离, 其净化效果优异。该法能准确地分离不同种类的黄 曲霉素, 检测速度快且定性与定量准确, 检测限低, 可作为仲裁法使用, 但 仪器设备价格昂贵, 前处理方法相对繁琐, 若用到免疫亲和柱则会使试样检 测费用增加, 对操作人员的身体健康仍存在一定的危害。

ELISA 法也是近年来研究开发出来的一种较为新颖的方法。其原理是根 据抗体和抗原之间特异性的免疫学反应, 最后用测定酶活力的方法来增加测 定的灵敏度。该方法检测速度快、对人体危害小、但重复性差、试剂寿命短、 需低温保存、假阳性概率较高、需要配置专门的酶标仪, 且对一些富含盐和 脂肪的试样需进行额外的处理。

毛细管电泳 (CE) 也是一种新发展起来的分析黄曲霉素的方法。该方法与 激光减弱荧光检测器(LIF)连用可很好地提高灵敏度。Wei 等用毛细管电泳激光减弱荧光检测器测定 $A_{F B} 、 A F B_{2} 、 A F G_{1}$ 和 $A F G_{2}$, 取得了较为理想的分 离效果, 其中对 $\mathrm{AFB}_{2}$ 的测定最为灵敏。但 $\mathrm{CE}$ 法的成本较高, 操作复杂, 不 适宜在试样检测中广泛应用。

IAC /SFB 法也是一种常用的国标方法。该方法的原理是利用各种黄曲霉 素的荧光特性差异用荧光光度计测定试样中黄曲霉素的含量。该方法对检测 人员身体健康无危害, 检测速度迅速, 灵敏度高, 适用于大量试样检测, 且定 量准确, 但检测费用较高, 需要配置专用设备, 且不能对单一的毒素进行检测。

金标试纸法, 实际就是一种固相免疫分析法。其原理是利用抗体与抗原 的特异性结合反应, 可一步检测黄曲霉素。该法可在 5 10 min 内完成对试样 中黄曲霉素的定性测定, 具有简单、快速的特点, 且无须其他仪器设备的配 合, 既可在实验室中进行检测, 也可在现场进行实地测定, 但是其检测的准 确度、精度有待进一步的研究。

生物传感器是使用固定化技术将具有分子识别能力的生物活性物质与物 理化学换能器结合, 可以用来探测生物体内外的环境化学物质或与之起特异 性交互作用后产生响应的一种装置。由于生物传感器具有选择性高、响应快、 
操作简单、携带方便和适合于现场检测等优点, 因此各国科研工作者正积极 探索研制新型生物传感器用于检测黄曲霉素。

据资料显示, 西南大学马良, 张宇昊等人[15]采用自行设计搭建的激光诱 导荧光(LIF) - 高效毛细管电泳(HPCE)检测平台, 建立 LIF-HPCE 法对食品中 的黄曲霉毒素 $\mathrm{B}_{1}\left(\mathrm{AFB}_{1}\right)$ 进行高灵敏度检测。 HPCE 的检测强度远高于 HPLC, 尤其是 $\mathrm{AFG}_{2}$ 和 $\mathrm{AFB}_{2}$ 。样品和标准品的 LIF-HPCE 图谱远远优于普通的色谱 峰, $\mathrm{AFB}_{1}$ 的苂光响应值非常敏感, 体现了 LIF-HPCE 高效和高灵敏度检测 $\mathrm{AFB}_{1}$ 的优势。

\section{5. 激光诱导荧光}

由荧光的发光原理可知, 分子荧光光谱与激发光源的波长无关, 只与荧 光物质本身的能级结构有关，所以可以根据苂光谱线对荧光物质进行定性分 析鉴别[24] [25]。

使用激光诱导荧光原理检测目标成分有两种方式，直接检测而和间接检 测 [24]。直接检测针对自发苂光的物质; 间接检测针对不发苂光或发苂光很弱 的物质。不管是直接测定, 还是间接测定, 一般的采用标准工作曲线法, 取 各种已知量的苂光物质, 配成一系列的标准溶液, 测定出这些标准溶液的苂 光强度, 然后给出荧光强度对标准溶液的浓度的工作曲线。在同样的仪器条 件下，测定未知样品的炎光强度，然后从标准工作曲线上查出未知样品的浓 度(即含量)。

与普通的荧光检测器一样, 激光诱导荧光检测器主要由光源、光学系统、 检测池和光检测元件组成, 二者最重要的区别是激光器 [26]。激光器是激光诱 导荧光检测器的重要组成部分，用脉冲激光为光源，采用时间分辨技术可消 除瑞利散射光(半径比光或其他电磁辐射的波长小很多的微小颗粒对入射光 束的散射)和拉曼散射光(光波在被散射后频率发生变化)对测定的干扰，同时 增加被测成分之间测定的选择性。以上这些特性使激光诱导荧光检测器的信 噪比大大增强, 显示出最高的灵敏度和较好的选择性。照射光越强, 被激发 到激发态的分子数越多, 测量时灵敏度越高, 一般由激光诱导荧光测量物质 的特性比由一般光源诱导苂光所测的灵敏度提高 2 10 倍[26]。

\section{6. 结论}

花生作为一种重要的粮油产品, 如何检测及控制花生中黄曲霉素的含量 很有现实意义。传统黄曲霉素检测方法大都采用化学或生物分析方法，检测 精度虽高，但检测时间长，不方便现场快速检测。从光谱分析角度看，黄曲 霉素具有光诱导荧光特性，在紫外线照射下，能发出蓝紫色、绿色的荧光。 因此可以通过检测光诱导的苂光来对黄曲霉素进行定性定量检测，对于污染 花生的甄别意义重大。

\section{Conflicts of Interest}

The authors declare no conflicts of interest. 


\section{References}

[1] 刘英, 胡建华, 刘春朝. 黄曲霉素毒理效应及检测方法[J]. 生物加工过程, 2013, 11(3): 83-88.

[2] 李秀缺, 张薇, 张爱菊, 张秋生. 花生中黄曲霉毒素的防控及去除方法 [J]. 食品 工程, 2010(2): 25-27+50.

[3] 陈冉. 花生中黄曲霉毒素降解技术研究[D]: [硕士学位论文]. 北京: 中国农业科 学院, 2013.

[4] 盛柏科. 黄曲霉素检验标准对中国花生类产品出口的影响及对策研究 [D]: [硕士 学位论文]. 南昌: 江西财经大学, 2010.

[5] 孙毅. 从黄曲霉毒素污染说开去[N]. 中国科学报, 2018-06-20(006).

[6] 安虹, 邹广迅. 黄曲霉素毒性效应机制的研究进展 [J]. 安徽农业科学, 2011, 39(24): 15007-15009+15012.

[7] 路子显, 伍松陵, 孙长坡. 黄曲霉素合成相关基因表达与环境因素的关系[J]. 生 物技术通报, 2010(11): 56-61+67.

[8］世界各国对黄曲霉素的控制标准[J]. 技术监督实用技术, 1996(5): 17.

[9] 马红岩, 马涛. 浅谈黄曲霉素的危害[J]. 山西农业(致富科技), 2007(6): 54.

[10] 王景彩, 董以爱. 重视黄曲霉素的危害[N]. 中国畜牧报, 2002-06-30(004).

[11] 刘英, 胡建华, 刘春朝. 黄曲霉素毒理效应及检测方法[J]. 生物加工过程, 2013, 11(3): 83-88.

[12] Clifford, J.I. and Rees, K.R. (1966) Aflatoxin: A Site of Action in the Rat Liver Cell. Nature, 209, 312-313. https://doi.org/10.1038/209312a0

[13] 陈靖文. 黄曲霉素的检测方法及预防措施[J]. 科学技术创新, 2019(20): 47-48.

[14] 殷爽, 陈振宜, 徐文杰, 陈娜, 刘书朋. 超低浓度黄曲霉素光电检测装置研制 [J]. 工业控制计算机, 2017, 30(8): 113-114.

[15] 杨静, 哈益明, 王锋. 高效液相色谱-串联质谱法检测花生中的黄曲霉毒素 B_1 [J]. 分析试验室, 2009, 28(6): 35-38.

[16] 邓欣, 朱志金金, 黄芳, 黄晓兰, 吴惠勤. 液相色谱-串联质谱法测定食用油中 4 种 黄曲霉素[J]. 广东化工, 2017, 44(15): 237-239.

[17] 何翠霞. 高效液相色谱在黄曲霉素检测中的应用[J]. 食品安全导刊, 2016(18): 116.

[18] 车畅. 黄曲霉素分析的几种方法及其比较[J]. 养殖技术顾问, 2010(3): 130.

[19] 赵宁, 郭玉梅, 姚妍妍, 许佳. 高效液相色谱法测定花生中黄曲霉素的含量 [J]. 科技信息, 2009(25): 44+29.

[20] Li, J.M., Liu, X.C., Li, X.Y., Ma, Q.X., Zhao, N., Zhang, Q.M., Guo, L. and Lu, Y.F. (2020) Investigation of Excitation Interference in Laser-Induced Breakdown Spectroscopy Assisted with Laser-Induced Fluorescence for Chromium Determination in Low-Alloy Steels. Optics and Lasers in Engineering, 124, Article ID: 105834. https://doi.org/10.1016/j.optlaseng.2019.105834

[21] 黄光琦. 黄曲霉素 B1 与 tRNA 的结合作用[J]. 中国药理学与毒理学杂志, 1993(3): 227-230.

[22] 马良, 张宇昊, 李培武. LIF-HPCE 法检测食品中的黄曲霉毒素 B1[J]. 食品科学, 2009, 30(10): 135-139.

[23] 李公喆. 黄曲霉素检验方法(普检法) [J]. 中国兽药杂志, 1983(4): 28-41.

[24] 梁锡辉, 区伟能, 任豪, 付治新. 激光诱导荧光检测技术 [J]. 激光与光电子学进 展, 2008(1): 65-72. 
[25] Peng, J., Lynen, F. and Sandra, P. (2000) Characterization of Inulins by Capillary Electrophoresis with Laser Induced Fluorescence Detection. Journal of High Resolution Chromatography, 23, 656-658.

https://doi.org/10.1002/1521-4168(20001101)23:11<656::AID-JHRC656>3.0.CO;2-Z

[26] 张玲玲, 陈媛, 王彩霞, 王春霞, 李国旗, 孙杰, 王健超, 贾锋. 便携式 $405 \mathrm{~nm}$ 激 光诱导荧光检测仪的研制和性能评估[J]. 应用激光, 2019, 39(6): 1035-1040.

\section{Appendix (Abstract and Keywords in Chinese) 花生中黄曲霉素研究现状综述}

摘要: 食品安全是饮食安全体系的重要一环, 与人们的身体健康息息相关。 花生作为我国重要的日常粮油产品, 确保其安全性非常重要, 但花生容易被 黄曲霉素污染。如何高效检测花生中黄曲霉素就显得尤为重要。本文对近年 来国内外花生中黄曲霉素的相关研究文献进行综述, 对其来源、危害和检测 方法等做出论述, 讨论了激光诱导荧光检测技术的优越性, 对未来的花生中 黄曲霉素检测提出一些建议。

关键词：黄曲霉素，花生，检测技术，激光诱导苂光 